\title{
ISOLATION AND CHARACTERIZATION OF A C-REPEAT BINDING FACTOR GENE FROM TEVANG-1 MAIZE CULTIVAR
}

\author{
Nguyen Thuy Linh ${ }^{1}$, Luu Han Ly ${ }^{1}$, Nguyen Thuy Duong ${ }^{1,2}$, Huynh Thi Thu Hue ${ }^{1,2, *}$ \\ ${ }^{1}$ Institute of Genome Research, VAST, Vietnam \\ ${ }^{2}$ Graduate University of Science and Technology, VAST, Vietnam
}

Received 24 April 2019, accepted 18 July 2019

\begin{abstract}
C-repeat binding factor $(\mathrm{CBF})$ proteins are transcription factors involved in plant response to abiotic stresses, especially low-temperature condition. In this research, a CBF3-coding gene was isolated from a cold-acclimation maize variety, Zea mays var. Tevang-1 and denoted as ZmCBF3tv. The isolated gene shared $96.49 \%$ homology with the B73-reference gene and had no intron in the coding sequence. By using bioinformatic tools, a number of variations in the nucleotide and amino acid sequences were identified. An alignment between ZmCBF3tv and other CBF/DREB1 proteins from various species revealed functional regions and typical features, such as nuclear localization signal (NLS), the AP2 DNA-binding domain, and acidic-amino-acidrich segments. Additionally, a phylogenetic analysis based on the AP2 domain showed that the maize $\mathrm{CBF} 3$ transcription factor had the highest similarity with that from rice and closely related to other CBF/DREB1 protein of monocots. The function of the ZmCBF3tv product is suggested to be a CBF/DREB1 transcription factor.
\end{abstract}

Keywords: CBF/DREB1, transcription factor, Tevang-1, maize, cold tolerance.

Citation: Nguyen Thuy Linh, Luu Han Ly, Nguyen Thuy Duong, Huynh Thi Thu Hue, 2019. Isolation and characterization of a C-repeat binding factor gene from Tevang-1 maize cultivar. Academia Journal of Biology, 41(3): 85-100. https://doi.org/10.15625/2615-9023/v41n3.13782.

*Corresponding author email: hthue@igr.ac.vn

(C2019 Vietnam Academy of Science and Technology (VAST) 


\section{INTRODUCTION}

Plant responses to abiotic stresses encompass the harmonization of different genes which encode regulatory and functional proteins. Among them, transcription factors (TF) have a central role in plant defense mechanisms that being activated upon stress then subsequently induce the expression of other stress-responsive genes. Therefore, analyzing and clarifying these TF genes' function are critical for understanding the plant response at the molecular level. In turn, it may contribute to creating enhanced stresstolerant crops by genetic engineering (Century et al., 2008). Hundreds of TF genes related to plant stress resistance have been isolated and characterized in many species. Most of TF genes are classified into several large families, such as AP2/ERF, NAC, MYC, bZIP, etc (Umezawa et al., 2006).

C-repeat binding factors (CBF), also referred to as dehydration responsiveelement binding factor 1 (DREB1) proteins are members of ERF subfamily of AP2/ERF superfamily. The CBF/DREB1 belonging to the A-1 subgroup are mainly participated in plant response to low temperature (Akhtar et al., 2012). $C B F$ genes have been found in a wide range of plants, such as AtCBFs from Arabidopsis thaliana (Gilmour et al., 2004), $B n C B F$ s from Brassica napus (Jaglo et al., 2001), CbCBF from Capsella bursa-pastoris (Wang et al., 2004), CfCBF3 from sweet pepper (Yang et al., 2011), EglCBFs from Eucalyptus (Nguyen et al., 2017; NavarreteCampos et al., 2017), $H v C B F 3$ from barley (Choi et al., 2002), $L p C B F 3$ from perennial ryegrass (Xiong \& Frei, 2006), OsCBF1 from rice (Choi et al., 2002), $P p C B F 3$ from bluegrass (Zhuang et al., 2015), $P a C B F$ from sweet cherry (Kitashiba et al., 2004), $S c C B F$ from rye (Jaglo et al., 2001), $L e C B F$ s from tomato (Zhang et al., 2004), TaCBFs from wheat (Babben et al., 2018), TmCBFs from Triticum monococcum (Miller et al., 2006), $V v C B F s$ from grapevine (Londo \& Garris, 2015), and $Z m C B F 3$ from maize (Wang et al., 2008).
CBF proteins contain several conserved domains including the DNA-binding domain $\mathrm{AP} 2$, the N-terminus nuclear localization signal (NLS), and the acidic domains present in C-terminus. CBF/DREB1 proteins are capable to bind to DNA regulatory elements called CRT (C-repeat)/DREB (dehydration responsive element) located at the promoter region of target genes. It has been reported that $\mathrm{CBF} / \mathrm{DREB} 1$ transcription factors enhance the cold, drought, and salinity tolerance of the transgenic plants. For instance, the transgenic Arabidopsis plants harboring $C B F 1$ gene showed a remarkable increase in abiotic-stress tolerance due to the high-level expression of cor 6.6, cor15a, cor47, and cor78 (Kasuga et al., 1999). Meanwhile, CBF3 induced the transcription of rd29A, rd17, cor6.6, cor15a, erd10, and kin1 and biochemical changes related to cold acclimation including total soluble sugars and proline accumulation (Gilmour et al., 2000). The same result was observed when introduce $C a C B F 3$ into tobacco plant (Yang et al., 2011). Recently, seven CBFs that significantly related to cold-tolerance were identified in wheat (Babben et al., 2018). The association analysis clarified the relationship between polymorphic sites located on the promoter and the coding sequence of $T a C B F s$ and cold-acclimation phenotype (Babben et al., 2018).

In this study, we isolated and characterized a $C B F$ homolog gene from Zea mays var. Tevang-1. The Tevang- 1 is local maize of Vietnam Northern mountainous area. This variety was reported to be able to tolerate drought and low-temperature condition, in other words, cold acclimation. Then, the molecular characteristics of $\mathrm{ZmCBF3tv}$ gene and its putative protein were analyzed using bioinformatic tools.

\section{MATERIALS AND METHODS}

\section{Plant materials and stress treatment}

Seeds of Z. mays var. Tevang- 1 were sown in soil in the greenhouse at $28^{\circ} \mathrm{C}$ under a photoperiod of 14-hours light and 10-hours dark. Plants at the 4-leaf stage were subjected 
to cold stress by placing in a $4^{\circ} \mathrm{C}$ refrigerator for 24,48 , and 72 hours. Leaf samples were collected and stored in the RNAlater ${ }^{\mathrm{TM}}$ Stabilization Solution (Invitrogen ${ }^{\mathrm{TM}}$, USA) for RNA extraction.

\section{RNA extraction and isolation of the ZmCBFtv gene}

Total RNA was extracted using GeneJET RNA Purification Kit (Thermo Scientific ${ }^{\mathrm{TM}}$, USA). Then, the cDNA was synthesized by USB ${ }^{\circledR}$ First-Strand cDNA Synthesis Kit for Real-Time PCR (Affymetrix Inc., USA). Based on the $Z m C B F 3$ sequence reported previously (Wang et al., 2008), specific primers for this gene were designed. The sequence containing CBF-coding region along with 5'UTR and 3'UTR segments were amplified from cDNA of Tevang-1 cultivar using the Forward primer (5'AACAGCAAGCTCAAGCAGTCAG -3') and the Reversed primer (5'TTGGGGTTGGCTGTCAAGCT -3'). Each $25 \mu \mathrm{L}$ RT-PCR reaction contained $12.5 \mu \mathrm{L}$ 2X Thermo Scientific DreamTaq PCR Master Mix, $1 \mu \mathrm{L}$ of $10 \mu \mathrm{M}$ each primer, $1 \mu \mathrm{L}$ of $\mathrm{c}$

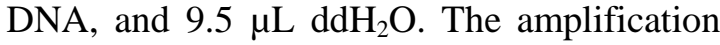
program was 4 minutes at $95^{\circ} \mathrm{C}$ followed by 30 cycles of 30 seconds at $95^{\circ} \mathrm{C}, 60$ seconds at $60^{\circ} \mathrm{C}$, and 60 seconds at $72^{\circ} \mathrm{C}$; then 5 minutes of $72^{\circ} \mathrm{C}$. The PCR product was purified by GeneJET PCR Purification Kit (Thermo Scientific $^{\mathrm{TM}}$, USA), then cloned into a pJET1.2 cloning vector following the manufacturer's instruction of CloneJET PCR Cloning Kit (Thermo Scientific ${ }^{\mathrm{TM}}$, USA).

\section{Molecular and bioinformatic analysis of ZmCBF3tv gene and putative protein}

The nucleotide composition of the isolated gene was obtained by sequencing the recombinant vector with pJET1.2 forward and reverse primers (5'- CGACTCACTATAGG GAGAGCGGC -3' and 5'- AAGAACATCG ATTTTCCATGGCAG -3', respectively) using the Big Dye Terminator kit (ABI Foster City, USA) on an Applied Biosystems ${ }^{\mathrm{TM}} 3500$ system. The sequencing results were edited and assembled by BioEdit program (http://www.mbio.ncsu.edu/BioEdit/-page2.ht $\mathrm{ml})$. The obtained sequence was annotated by BLASTN and BLASTX tools against appropriate collection database on https://www.ncbi.nlm.nih.gov/. Then, the isolated gene was compared to the reference gene from B73 RefGen_v4 on http://maizegdb.org. The deduced amino acid sequence of $Z m C B F 3 t v$ was translated using the ExPASy tool (https://web.expasy.org/ translate/). Physical and chemical characteristics of the putative $Z m C B F 3 t v$ protein were estimated by the ProtParam web tool (https://web.expasy.org/protparam/). The relationship of $\mathrm{ZmCBF} 3 \mathrm{tv}$ and other transcription factors of the ERF/AP2 family was carried out by comparing the AP2 domain sequences. The protein alignment was proceeded by BioEdit and then manually edited to final consistency. The phylogenetic tree was constructed using the neighborjoining method by MEGAX software (https://megasoftware.net/). All ERF/AP2 protein sequences were retrived from GenBank and listed in table 1.

Table 1: ERF/AP2 transcription factors and their accession numbers

\begin{tabular}{|c|c|c|}
\hline Species & ID & Abbreviation \\
\hline \multicolumn{3}{|c|}{ CBF/DREB1 transcription factors } \\
\hline Dicots & & \\
\hline Brassica napus & AAL38243.1 & $\mathrm{Bn}$ \\
\hline Brassica rapa & AHL44965.1 & $\mathrm{Br}$ \\
\hline Brassica oleracea & AAQ02702.1 & Bo \\
\hline Ageratina adenophora & AIT39763.1 & $\mathrm{Aa}$ \\
\hline Nicotiana tabacum & ACE73693.1 & $\mathrm{Nt}$ \\
\hline Arabidopsis thaliana & ABV27151.1 & Ath \\
\hline
\end{tabular}




\begin{tabular}{|c|c|c|}
\hline Malcomia scorpioides & AFZ94953.1 & Msc \\
\hline Arabis pumila & ABA42927.1 & Ap \\
\hline Iris lactea & AAZ57434.1 & Il \\
\hline Capsella bursa-pastoris & AAR26658.1 & $\mathrm{Cb}$ \\
\hline Capsicum annuum & AAQ88400.1 & $\mathrm{Ca}$ \\
\hline Capsicum frutescens & ADM73296.1 & $\mathrm{Cf}$ \\
\hline Solanum melongena & AWV55520.1 & $\mathrm{Sm}$ \\
\hline Solanum tuberosum & NP 001274894.1 & St \\
\hline Solanum lycopersicum & AAS77819.1 & $\mathrm{Sl}$ \\
\hline Solanum pimpinellifolium & ASK51844.1 & $\mathrm{Sp}$ \\
\hline Hevea brasiliensis & AAY43213.1 & $\mathrm{Hb}$ \\
\hline Eucalyptus globulus & AQX36212.1 & Egl \\
\hline Santalum album & ARM20323.1 & $\mathrm{Sa}$ \\
\hline Morus alba & AFQ59977.1 & $\mathrm{Ma}$ \\
\hline Medicago truncatula & AGR40678.1 & $\mathrm{Mt}$ \\
\hline Glycine $\max$ & NP 001235507.1 & $\mathrm{Gm}$ \\
\hline Glycine soja & AAV66464.1 & Gs \\
\hline Eucalyptus gunnii & APG53558.1 & Egu \\
\hline Populus trichocarpa & $\mathrm{ABO} 48365.1$ & $\mathrm{Pt}$ \\
\hline Malus domestica & ART85560.1 & $\mathrm{Md}$ \\
\hline Malus sieversii & ARO50175.1 & Msi \\
\hline Prunus avium & BAD27123.1 & $\mathrm{Pa}$ \\
\hline Vitis labrusca & AIL00737.1 & Vl \\
\hline Vitis vinifera & AIL00738.1 & $\mathrm{Vv}$ \\
\hline \multicolumn{3}{|l|}{ Monocots } \\
\hline Bambusa emeiensis & AFH68054.1 & $\mathrm{Be}$ \\
\hline Aegilops tauschii & AVO65154.1 & Ata \\
\hline Secale cereale & AAL35760.1 & $\mathrm{Sc}$ \\
\hline Festuca arundinacea & AAQ98965.1 & $\mathrm{Fa}$ \\
\hline Aegilops biuncialis & CBX87017.1 & $\mathrm{Ab}$ \\
\hline Triticum monococcum & AAY32553.1 & $\mathrm{Tm}$ \\
\hline Triticum aestivum & AWT24536.1 & $\mathrm{Ta}$ \\
\hline Hordeum vulgare & AEP32120.1 & $\mathrm{Hv}$ \\
\hline Avena sativa & CAJ21277.1 & As \\
\hline Brachypodium distachyon & AFD96409.1 & $\mathrm{Bd}$ \\
\hline Lolium perenne & AAX57275.1 & Lp \\
\hline Poa pratensis & ALG05365.1 & $\mathrm{Pp}$ \\
\hline Sorghum bicolor & AAX28960.1 & $\mathrm{Sb}$ \\
\hline Oryza sativa & AAG59619.1 & Os \\
\hline Zea mays & XP 008670333.1 & $\mathrm{Zm}$ \\
\hline \multicolumn{3}{|c|}{ DREB2 transcription factors } \\
\hline Glycine $\max$ & AAQ57226.1 & Out.Gm \\
\hline Sorghum bicolor & ABD66654.1 & Out.Sb \\
\hline Zea mays & NP 001292873.1 & Out.Zm \\
\hline
\end{tabular}




\section{RESULTS AND DISCUSSION}

\section{Isolation of $\mathrm{ZmCBF} 3 t v$ gene from Tevang-1 maize}

The total RNA samples extracted from leaves of one, two, and three-day cold treatment plant were checked by electrophoresis on $0.8 \%$ agarose gel and then used as templates for cDNA synthesis experiment (Fig. 1a). The RT-PCR analysis was carried out using the specific primer pair and condition as described above. As the result, DNA bands which were nearly $0.9 \mathrm{~kb}$ in length were observed in the 24 and 48 hours cold-treated samples (Fig. 1b). The PCR products were purified and then inserted into the cloning vector - pJET1.2. $\mathbf{a}$

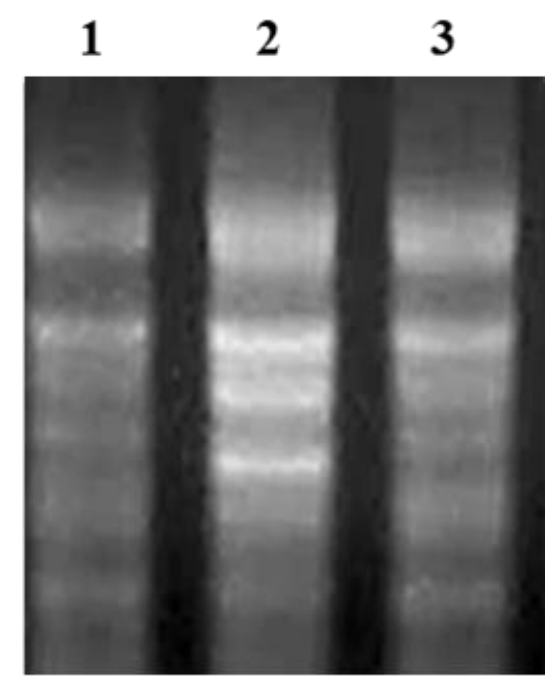

b

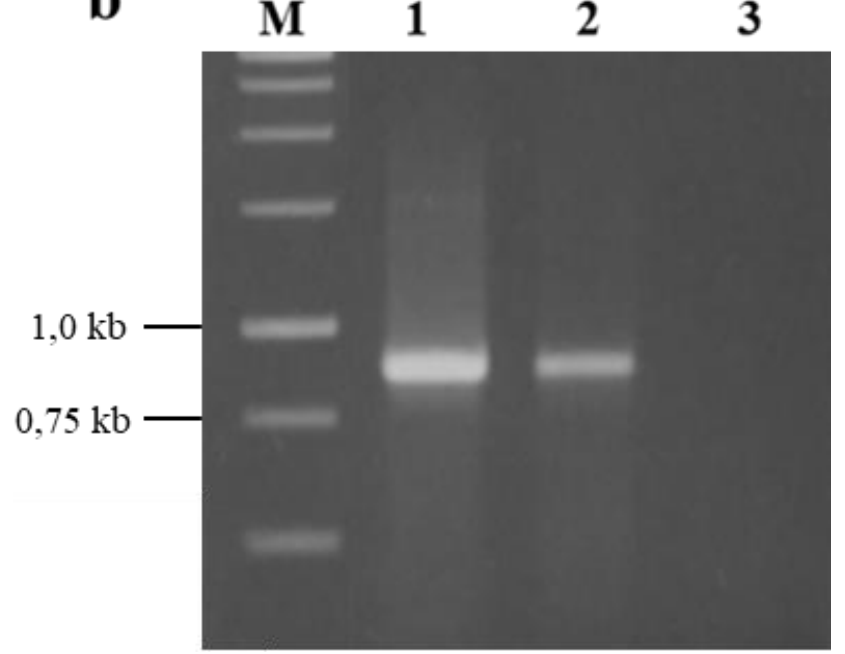

Figure 1. Isolation of ZmCBF3tv gene from Tevang-1 maize: a. Result of total RNA extraction. 1-3: Total RNA from maize plants after 24, 48, and 72 hours under low-temperature condition, respectively; b. Result of RT-PCR reaction. M: GeneRuler $1 \mathrm{~kb}$ DNA Ladder (Thermo Scientific, USA). 1-3: PCR products using cDNA templates of 24, 48, and 72-hours cold-treated samples, respectively

Analysis of nucleotide sequence of ZmCBF3tv gene from Tevang-1 maize

Sequencing of the inserted fragment in the recombinant vector $\mathrm{pJET} 1.2$ revealed that the isolated cDNA had 908 nucleotides (Fig. 2). A BLASTN analysis on GenBank showed that this sequence shared similarities to many genes encoding CRT/DRE binding factor protein from maize, sorghum, barley, and panicgrass. The isolated sequence had the highest homology (96.49\%) to PREDICTED: Zea mays dehydration-responsive elementbinding protein $1 \mathrm{H}$ (LOC103647602), mRNA (ID: XM_008672111.3) from Z. mays var. $B 73$ followed by $Z m C B F 3$ mRNA (95.86\%) (ID: AY964718.1) of Z. mays var. Qi319
(Wang et al., 2008). Besides, by using BLASTX tool against the UniProtKBTrEMBL database and non-redundant proteins, it is confirmed that the query carried the genetic information of a TF belonging to the ERF/AP2 family. Therefore, the isolated cDNA from Tevang-1 maize cultivar was denoted as ZmCBF3tv. Additionally, the genomic DNA sequence that corresponded to the ZmCBF3tv cDNA was also identified through a BLAST search on http://www.maizegdb.org/. According to the result, $C B F 3$ gene was located on chromosome 2 and contained no intron inside the coding sequence as other CBF/DREBI genes (Akhtar et al., 2012). 


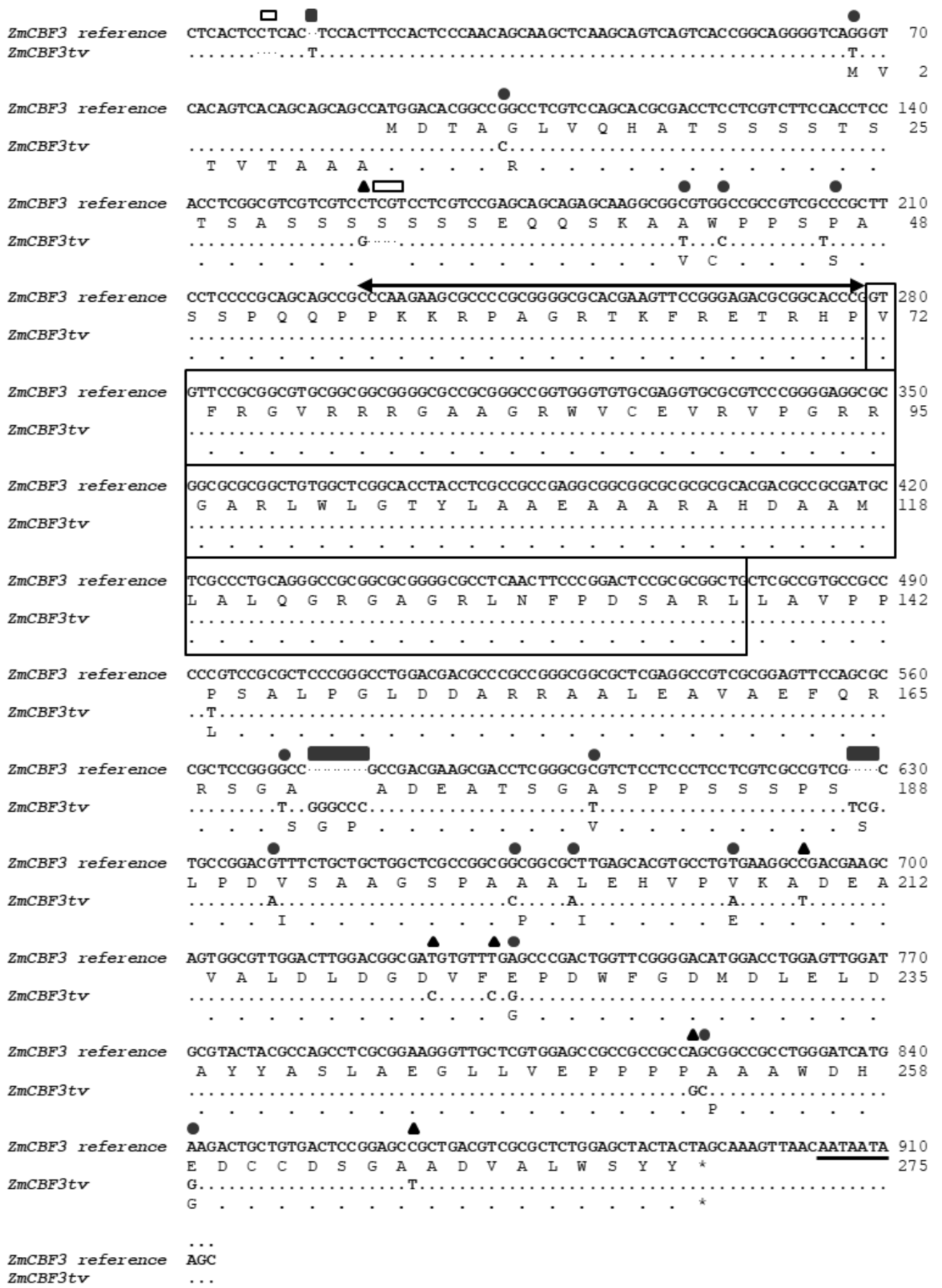

Figure 2. The $Z m C B F 3 t v$ sequence and its putative protein: The $Z m C B F$ reference sequence originated from B73 maize that downloaded from http://www.maizegdb.org/. The putative protein sequences were translated by ExPASy tool. The AP2 DNA-binding domain was enclosed inside boxes. A double-headed arrow demonstrated the nuclear localization signal

(NLS) and the polyadenylated signal was underlined. Unfilled and filled rectangles represented deletion and insertion mutation, respectively. Triangles and dots showed nucleotide subtitutions including synonymous mutation and missense mutation, respectively 
The 908 bp ZmCBF3tv isolated from $Z$. mays var. Tevang- 1 contained an entire coding region and part of 5' and 3'UTR in which polyadenylated signal was underlined (Fig. 2). A nucleotide alignment confirmed that the ZmCBF3tv shared $96.49 \%$ homology with the $\mathrm{ZmCBF3}$ sequence from $\mathrm{B} 73$ reference maize (2:200398600-200397688). In the meantime, a number of variations in the coding region and 5'UTR region were identified. As shown in Fig. 2, variations dispersed at 5' end and occurred more frequently at 3' end of the coding region. However, the segment defining the DNAbinding domain in the TF was conserved. Among such variations, one deletion of TCG codon at position 159 and two insertion mutations including GGCCCG at position 574 and TCG at position 627 increased the nucleotide number of ZmCBF3tv gene by six. Noticeably, one substitution mutation happened at position 67 which formally was the 5'UTR of $\mathrm{ZmCBF3}$ from B73 maize created a new ATG codon. In fact, the B73origin $\mathrm{ZmCBF3}$ consists of an open reading frame sequence expanding 804 bp segment. The appearance of an upstream ATG codon probably led to the addition of 24 nucleotides to the beginning of $Z m C B F 3 t v$ coding region. Nevertheless, these variations did not affect the reading frame of $\mathrm{ZmCBF} 3 t v$ protein.

\section{Sequence analysis of $\mathrm{ZmCBFtv}$ protein in silico}

The deduced ZmCBF3tv protein contained 274 amino acids and was rich in Ala (17.2\%), Ser (12.0\%), and Pro (9.5\%). Besides, the protein contained a low number of Asn and Ile, with the figure of one and two residues, respectively. The molecular weight of $\mathrm{ZmCBF} 3 \mathrm{tv}$ was predicted at $28.6 \mathrm{kDa}$ with the theoretical isoelectric point of 5.58. The instability index and GRAVY were calculated of 68.20 and -0.314 , respectively, suggesting that the ZmCBF3tv protein had an unstable and non-polar nature.

By comparing the $\mathrm{ZmCBF} 3 \mathrm{tv}$ protein to the homolog one originated from B73 maize, we found many changes in the composition of the polypeptide (Fig. 2). In term of protein length, due to the appearance of an ATG codon at position 67 and three InDel mutations, the $\mathrm{ZmCBF} 3 \mathrm{tv}$ protein was 10 amino acids longer than the $\mathrm{ZmCBF} 3 \mathrm{TF}$ of B73 maize. By ways of illustration, an additional MVTVTAAA segment was detected at the most N-terminus while additional Gly-Pro and Ser residues were observed at position 170 and 188, respectively. On the other hand, the $7 x$ Ser cluster was replaced by a $6 x$ Ser sequence as a TCG codon located at position 159 was removed from ZmCBF3tv coding region. Furthermore, 14 nonsynonymous substitutions including eight transversion mutations and six transition mutations also impact the amino acid content of ZmCBF3tv. For instances, $102 \mathrm{G}>\mathrm{C}, 189 \mathrm{C}>\mathrm{T}$, and $733 \mathrm{~A}>\mathrm{G}$ caused the transition of G13R, A42V, and E223G, respectively (Fig. 2).

The alignment of $\mathrm{ZmCBF} 3$ tv with other CBF/DREB1 proteins from different species revealed functional regions and "signature" motifs of CBF3 proteins (Fig. 3). Because of being a $\mathrm{TF}$, the putative $\mathrm{ZmCBF}$ tv protein had a basic-amino-acid-rich sequence of PKKRPAGRTKFRETRHP as a nuclear localization signal (NLS) that help the protein entered the nucleus. After the NLS segment, the AP2 DNA-binding domains of AP2/ERF superfamily expand to a sequence of about 60 amino acids (Fig. 3). Additionally, several conserved regions rich in D and $\mathrm{E}$ and LWSY motif were identified near the C-terminus of ZmCBF3tv protein. Nevertheless, besides the high similarity between functional regions, the composition and structure of CBF/DREB1 proteins are quite diverse among species (Fig. 3).

All TFs belonging to AP2/ERF superfamily contain the AP2 motif that highly conserved within the family. The AP2 domains were analyzed further and used for phylogenetic analysis (Fig. 4, 5). The AP2 sequence consisted of two local regions: YRG and RAYD. While the 20-amino-acid YRG region that was predicted to be important to the DNA-binding activity rich in basic and hydrophobic residues, the RAYD region 
contained about 40 amino acids contributing to amphipathic property was proposed to mediate protein-protein interaction (Okamuro et al., 1997). Therefore, the AP2 DNAbinding domain was composed of three antiparallel $\beta$-sheets and one $\alpha$-helix. The alignment of AP2 domain in CBF/DREB1 TFs from Z. mays var. Tevang- 1 and other species led to a consistent result with previous studies. For instance, a number of highly conserved amino acids, in which, some are critical to DNA specificity-binding and activation activity, such as Arg $(6,8,27)$, Trp $(14,29)$, Val (15), and Glu (17) (Allen et al., 1998) were noticed. These residues were remain unchanged in $\mathrm{ZmCBF} 3 \mathrm{tv}$, suggesting that the C-repeat binding factor from Tevang1 might have the same target gene and bind to the cis-element on such gene promoter with the same affinity in comparison to other CBF/DREB1 proteins.

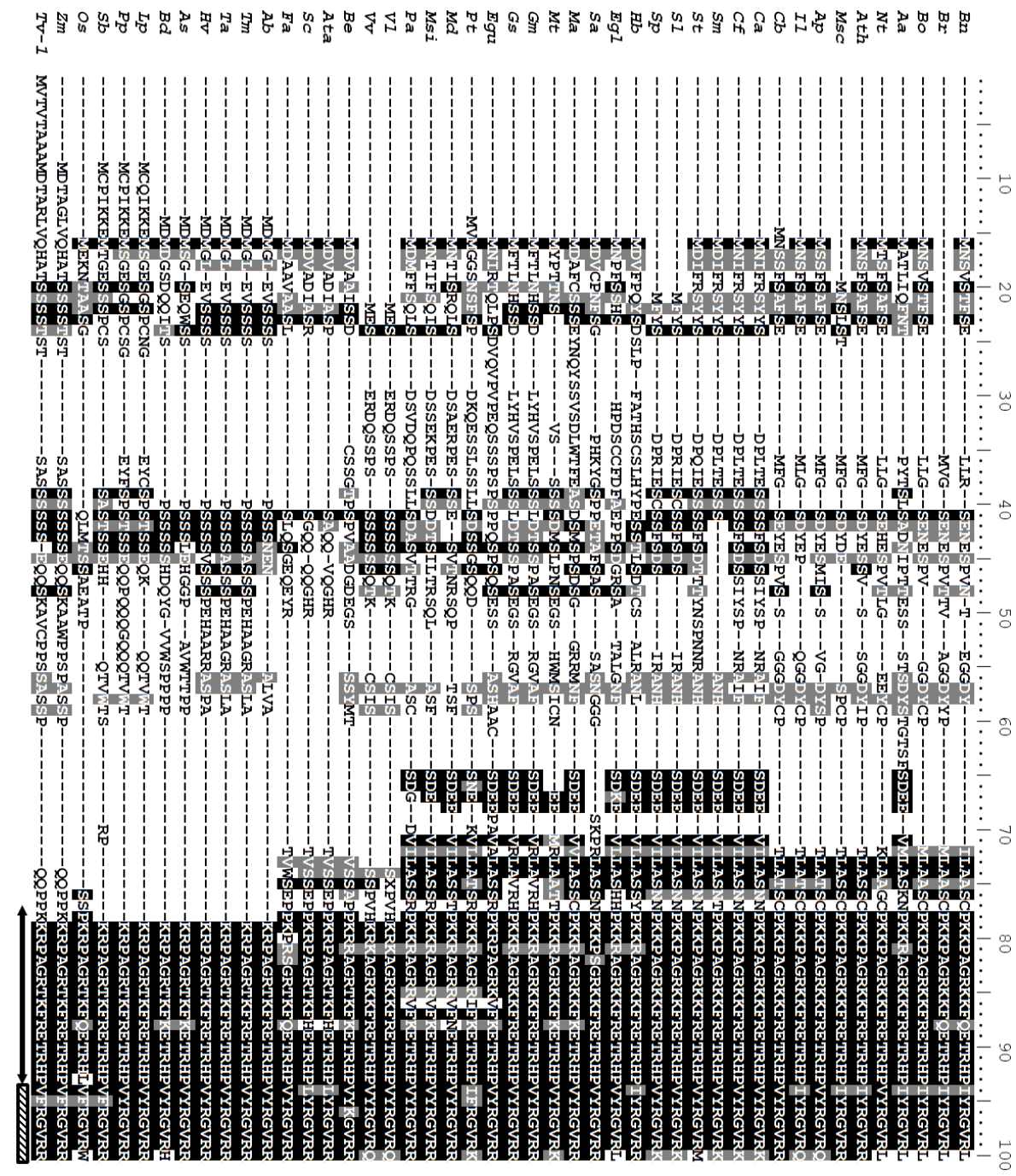

Figure 3. Amino acid sequence alignment of the putative CBF/DREB1 TFs of Tevang-1 maize (Tv-1) and those from various species. The nuclear localization signal (NLS) was demonstrated by a double-headed arrow. The DNA-binding domain was presented by rectangle box filled with upward diagonal pattern. Highly and moderately similar residues across species were shade in black and gray, respectively 


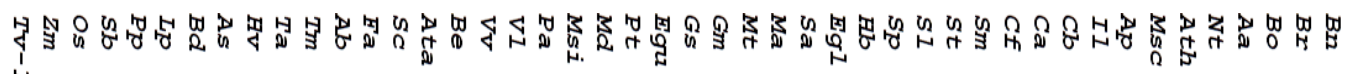

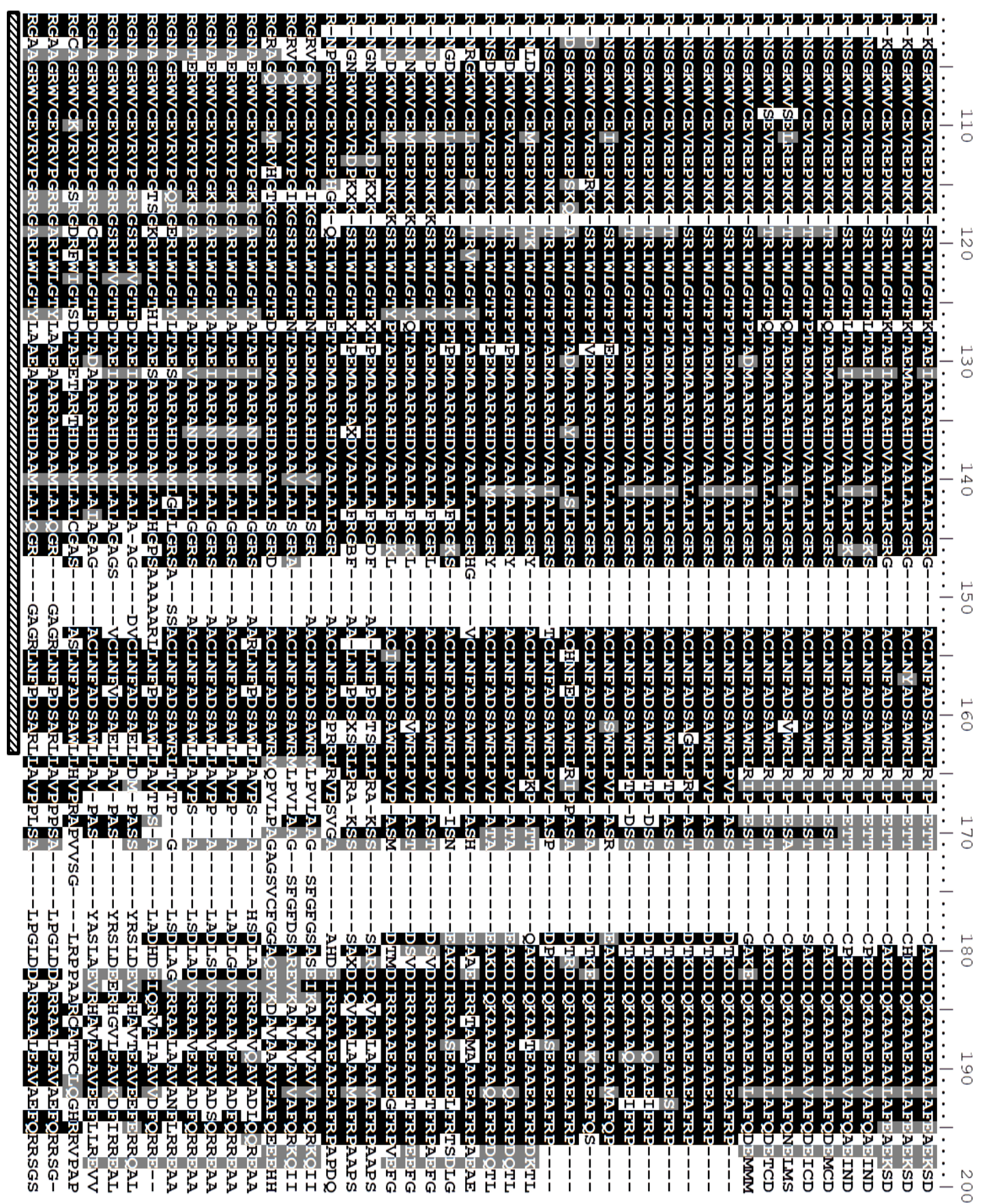

Figure 3. Amino acid sequence alignment of the putative CBF/DREB1 TFs of Tevang-1 maize (Tv-1) and those from various species. The nuclear localization signal (NLS) was demonstrated by a double-headed arrow. The DNA-binding domain was presented by rectangle box filled with upward diagonal pattern. Highly and moderately similar residues across species were shade in black and gray, respectively (next) 


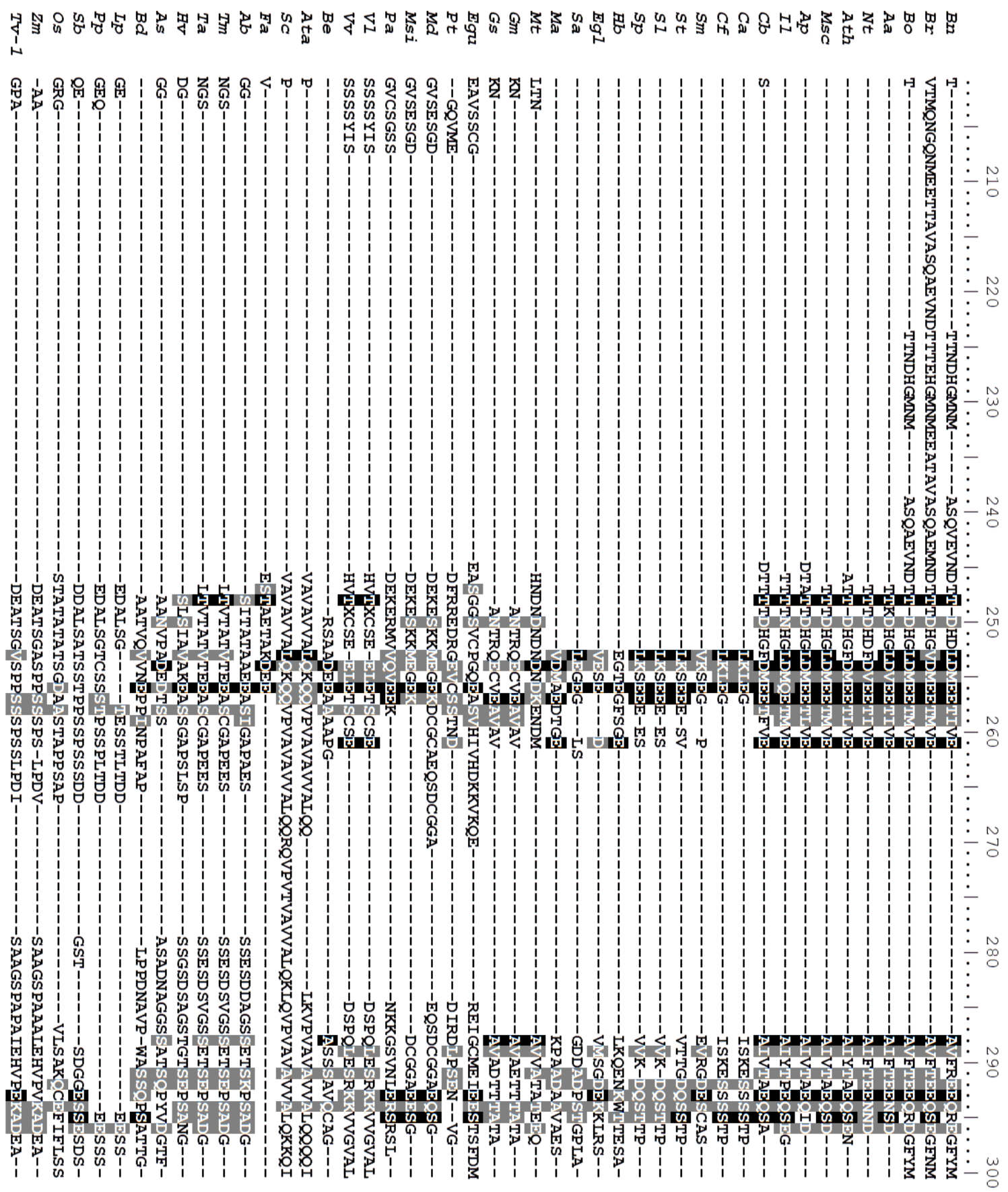

Figure 3. Amino acid sequence alignment of the putative CBF/DREB1 TFs of Tevang-1 maize (Tv-1) and those from various species. The nuclear localization signal (NLS) was demonstrated by a double-headed arrow. The DNA-binding domain was presented by rectangle box filled with upward diagonal pattern. Highly and moderately similar residues across species were shade in black and gray, respectively (next) 


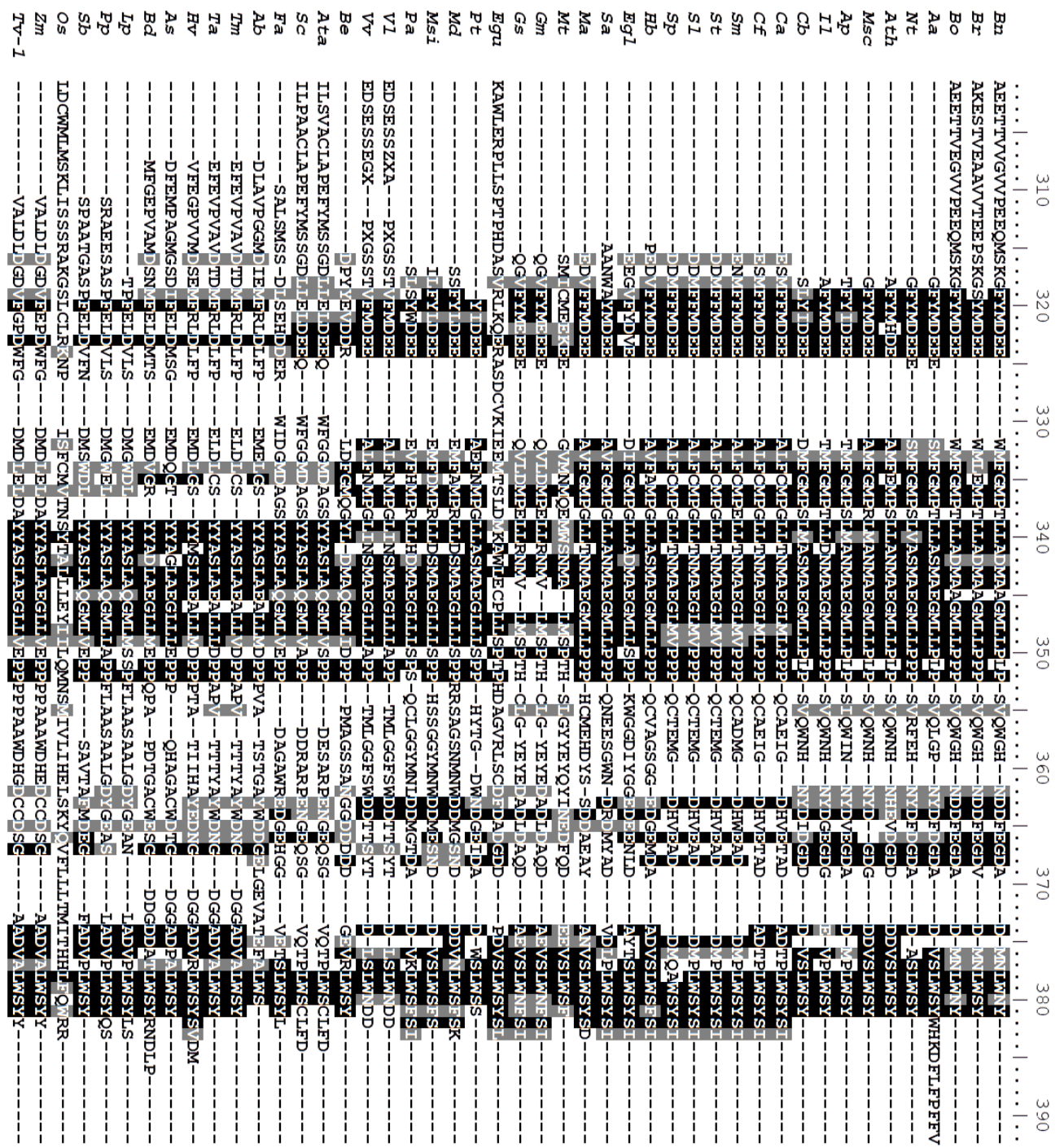

Figure 3. Amino acid sequence alignment of the putative CBF/DREB1 TFs of Tevang-1 maize (Tv-1) and those from various species. The nuclear localization signal (NLS) was demonstrated by a double-headed arrow. The DNA-binding domain was presented by rectangle box filled with upward diagonal pattern. Highly and moderately similar residues across species were shade in black and gray, respectively (next)

Whereas, there were several differences in amino acid content of the DNA-binding domain between the monocots and dicots CBF/ DREB1 proteins. For instance, Glu (20) was observed in almost dicot plants except for Vitis spp. contain Asp (20) while in monocots, the position was replaced by Val (20) in spite of B. emeiensis had Glu (20) (Fig. 4). Other moderately conserved positions in dicots were Lys (13), Asn (22), and Ile (28), but those in monocot were Arg (13), Gly (22), and Leu (28), respectively. Because of originating from maize, the ZmCBF3tv contained such monocots-typical residues. Noticeably, in the position 35 , the CBF/DREB1 transcription factor from $Z$. mays including B73 and Tevang-1 maize had Ala residue instead of Thr as those from other plants (Fig. 4). It was also identified that the DVAA (44) and DSAWR (66) motif were highly conserved 
among dicots. Meanwhile, most of the monocots including Tevang-1 contained
DAA(M/V) motif and DSAWL, respectively (Fig. 4).

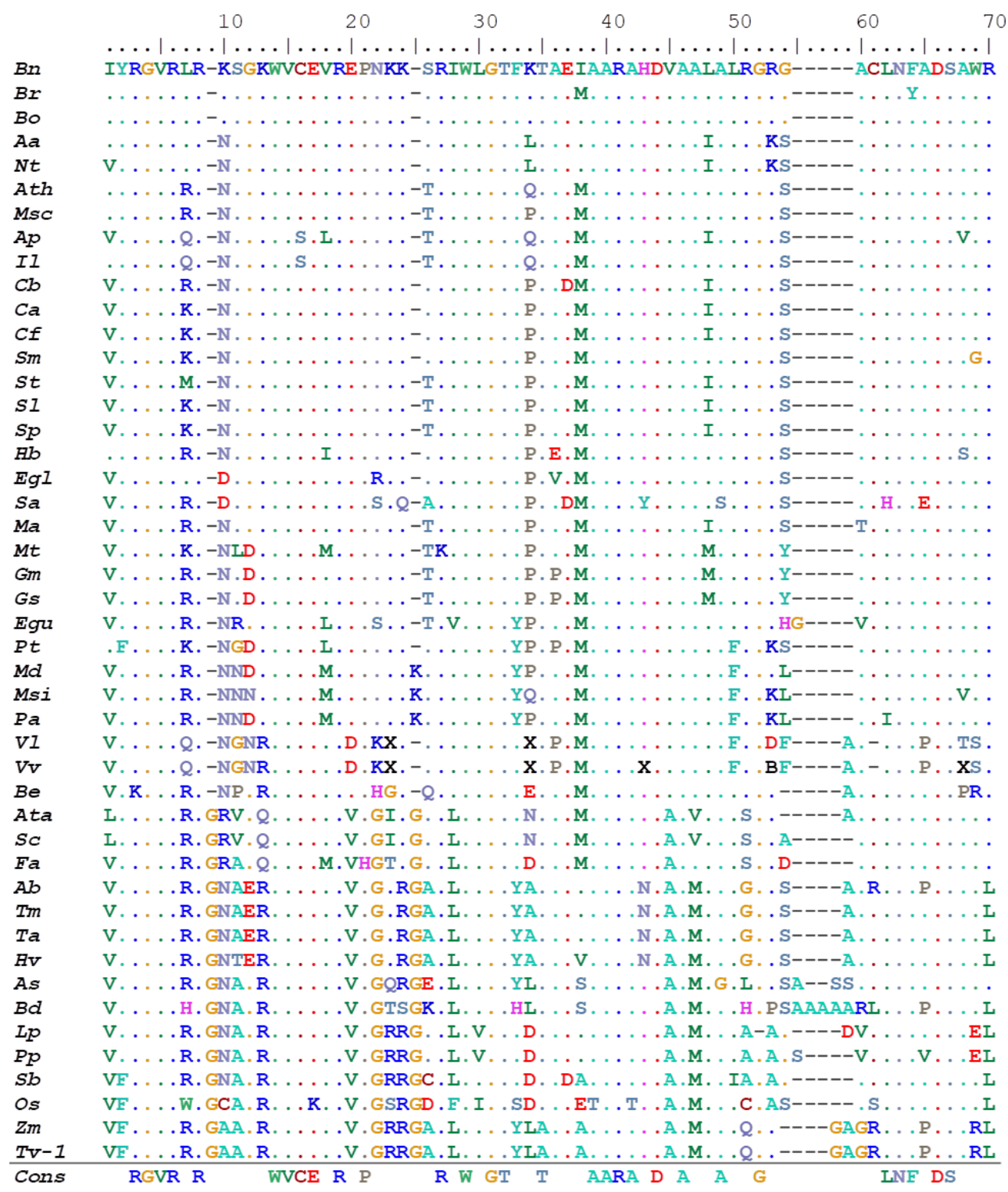

Figure 4. The comparison of the AP2 domain sequence between CBF/DREB1 transcription factors from various species. The Cons, or consensus sequence shown underneath was created using BioEdit software

Phylogenetic analysis of $\mathrm{ZmCBFtv}$ proteins

A phylogenetic analysis based on the AP2 domain sequence was carried out to clarify the phylogenetic relationship between ZmCBF3tv and other CBF/DREB1 TFs from diverse species. We compared the amino acid similarities in 48 proteins from 44 species, in which three DREB2 proteins served as outgroup samples. As shown in Fig. 5, the CBF/DREB1 transcription factors were well separated into two groups from monocots and dicots with the exception of B. emeiensis. The 
ZmCBF3tv shared the highest homology with one from $O$. sativa and had close relationships with those from monocots including barley, sorghum, wheat, etc (Fig. 5). The result was in agreement with the alignment of the AP2 domain (Fig. 4).

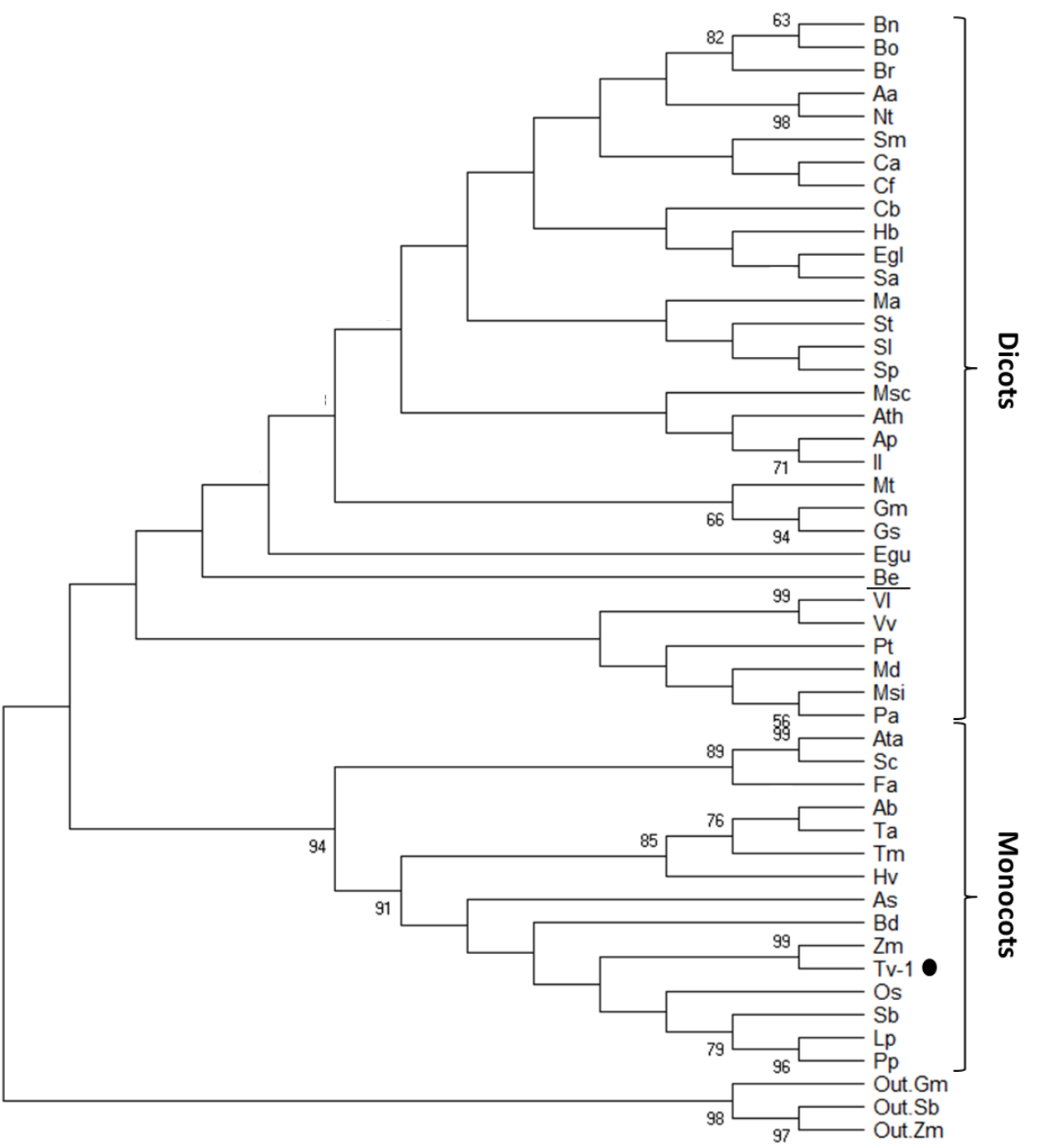

Figure 5. The phylogenetic tree base on AP2 sequence of CBF/DREB1 transcription factors from different species

CBF/DREB1 TF genes were induced by various abiotic stresses, such as lowtemperature condition. However, the expression pattern of CBF/DREB1 genes to stress were varied upon species. Wang et al. (2008) reported that the $Z m C B F 3$ of $Z$. mays var. Qi319 was strongly induced after 48 hours of cold stress. Nonetheless, $B j D R E B 1 B$ gene expressed immediately when $B$. juncea plants confronting to stresses - in other words after 20-minute treatment, and reached the highest transcription level between 2 and 12 hours (Cong et al., 2008). In this study, ZmCBF3tv gene was expressed after one and two days under $4^{\circ} \mathrm{C}$, in which the expression level at time-point of 24-hours was higher. However, there were no transcripts of ZmCBF3tv observed when using a longer cold-exposing time (72 hours) (Fig. 1). 
The alignment of $\mathrm{ZmCBF3}$ locus between Tevang-1 and B73 maize revealed 25 genetic variations $(3.51 \%)$ including 20 substitutions and five InDels upon a segment of $908 \mathrm{bp}$ long (Fig. 2). The percentage of variation occurred in $C B F / D R E B 1$ loci were analyzed in many plants and different according to genes and species. In wheat, the ratio of polymorphic sites to the coding sequence length of TaCBFs ranged from $0.00 \%$ to $3.54 \%$ (Babben et al., 2018). Meanwhile, a research examining 87 different sequences of $V v C B F 3$ showed $8.9 \%$ variation in the coding region (Londo \& Garris, 2014). Although $3.51 \%$ of $\mathrm{ZmCBF3}$ sequence contained genetic differences between two varieties (B73 and Tevang-1), this figure was not representative for maize due to the limitation in sample collection and the data size as only two accessions were included in the analysis. Nevertheless, the changes in the 5'UTR and the coding sequence of $Z m C B F 3 t v$ gene did alter the amino acid composition of $\mathrm{C}$-repeat binding factor. The changes in ZmCBF3tv polypeptide sequence might play a role in enhancing the cold-tolerance phenotype observed in Z. mays var. Tevang- 1 .

The study of putative CBF/DREB1 proteins from Tevang-1 maize and other species using bioinformatic tools suggested that ZmCBF3tv was a typical CBF/DREB1 $\mathrm{TF}$ that could induce the expression of various stress-responsive genes due to the presence of many functional and "signature" regions (Fig. 3, 4). Additionally, the putative ZmCBF3tv protein had a close phylogenetic relationship to other monocots and had the highest similarity to one from rice (Fig. 5). To clarify the function of the ZmCBF3tv transcription factor, it is necessary to conduct further experimental studies. Still, the results from this in sillico analysis of the putative protein gave clues on the ZmCBF3tv activity.

\section{CONCLUSIONS}

In this research, we isolated from Tevang1 maize variety a 908 bp cDNA called ZmCBF3tv that was $96.49 \%$ homology to the reference genome B73. The isolated fragment consisted of many variations in the 5'UTR and coding sequences. Furthermore, the putative $\mathrm{ZmCBF} 3 \mathrm{tv}$ protein had the conserved DNA-binding domain - AP2 and some signature regions such as NLS and C-terminus acidic domains. In addition, the $\mathrm{ZmCBF} 3 \mathrm{tv}$ had the close phylogenetic relationship with other CBF/DREB1 transcription factors from monocots. Therefore, it was implemented that the $Z m C B F 3 t v$ originated from $Z$. mays var. Tevang-1 carried the genetic information coding for a cold-response C-repeat binding factor.

Acknowledgement: This work was funded by Vietnam Ministry of Agriculture and Rural Development (MARD) under Grant No. 16HĐ/KHCN-VP for the period 2014-2018.

\section{REFERENCES}

Akhtar M., Jaiswal A., Taj G., Jaiswal J. P., Qureshi M. I., Singh N. K., 2012. DREB1/CBF transcription factors: their structure, function and role in abiotic stress tolerance in plants. J. Genet., 91(3): 385-395.

Allen M. D., Yamasaki K., Ohme-Takagi M., Tateno M., Suzuki M., 1998. A novel mode of DNA recognition by a betasheet revealed by the solution structure of the GCC-box binding domain in complex with DNA. EMBO J., 17: 5484-5496.

Babben S., Schliephake E., Janitza P., Berner T., Keilwagen J., Koch M., AranaCeballos F. A., Templer S. E., Chesnokov Y., Pshenichnikova T., Schondelmaier J., Borner A., Pillen K., Ordon F., Perovic D., 2018. Association genetics studies on frost tolerance in wheat (Triticum aestivum L.) reveal new highly conserved amino acid substitutions in $C B F-A 3, C B F$ $A 15, V R N 3$ and $P P D 1$ genes. $B M C$ Genomics, 19(1): 409.

Century K., Reuber T. L., Ratcliffe O. J., 2008. Regulating the regulators: the future prospects for transcription-factor-based agricultural biotechnology products. Plant Physiol., 147: 20-29.

Choi D. W., Rodriguez E. M., Close T. J., 2002. Barley Cbf3 gene identification, 
expression pattern, and map location. Plant Physiol., 129(4): 1781-1787.

Cong L., Chai T. Y., Zhang Y. X., 2008. Characterization of the novel gene BjDREB1B encoding a DRE-binding transcription factor from Brassica juncea L. Biochem. Biophys. Res. Commun., 371(4): 702-706.

Gilmour S. J., Fowler S. G., Thomashow M. F., 2004. Arabidopsis transcriptional activators $\mathrm{CBF} 1, \mathrm{CBF} 2$, and $\mathrm{CBF} 3$ have matching functional activities. Plant Mol. Biol., 54: 767-781.

Jaglo K. R., Kleff S., Amundsen K. L., Zhang X., Haake V., Zhang J. Z., Deits T., Thomashow M. F., 2001. Components of the Arabidopsis C-repeat/dehydrationresponsive element binding factor cold response pathway are conserved in Brassica napus and other plant species. Plant Physiol., 127: 910-917.

Kasuga M., Liu Q., Miura S., YamaguchiShinozaki K., Shinozaki K., 1999. Improving plant drought, salt, and freezing tolerance by gene transfer of a single stress inducible transcriptionfactor. Nat. Biotechnol., 17: 287-291.

Kitashiba H., Ishizaka T., Isuzugawa K., Nishimura K., Suzuki T., 2004. Expression of a sweet cherry DREB1/CBF ortholog in Arabidopsis confers salt and freezing tolerance. J. Plant Physiol., 161(10): 1171-1176.

Liu Q., Kasuga M., Sakuma Y., Abe H., Miura S., Yamaguchi-Shinozaki K., Shinozaki K., 1998. Two transcription factors, DREB1 and DREB2, with an EREBP/AP2 DNA binding domain separate two cellular signal transduction pathways in drought- and low-temperature responsive gene expression, respectively, in Arabidopsis. Plant Cell, 10: 391-406.

Londo J. P., Garris A. J., 2015. Genetic and protein sequence variation of CBF1-4 in cold hardy wild grapevine germplasm. VITIS, 53(4): 201-206.
Miller A. K., Galiba G., Dubcovsky J., 2006. A cluster of $11 \mathrm{CBF}$ transcription factors is located at the frost tolerance locus Fr-A m 2 in Triticum monococcum. Mol. Genet. Genomics, 275(2): 193-203.

Navarrete-Campos D., Le-Feuvre R., Balocchi C., Valenzuela S., 2017. Overexpression of three novel CBF transcription factors from Eucalyptus globulus improves cold tolerance on transgenic Arabidopsis thaliana. Trees, 31(3): 1041-1055.

Nguyen H. C., Cao P. B., San-Clemente H., Ployet R., Mounet F., Ladouce N., Harvengt L., Marque C., Teulieres C., 2017. Special trends in CBF and DREB2 groups in Eucalyptus gunnii vs Eucalyptus grandis suggest that $\mathrm{CBF}$ are master players in the trade-off between growth and stress resistance. Physiol. Plant., 159(4): 445-467.

Okamuro J. K., Caster B., Villarroel R., Van Montagu M., Jofuku K. D., 1997. The AP2 domain of APETALA2 defines a large new family of DNA binding proteins in Arabidopsis. Proc. Natl. Acad. Sci. USA, 94: 7076-7081.

Umezawa T., Fujita M., Fujita Y., Yamaguchi-Shinozaki K., Shinozaki K., 2006. Engineering drought tolerance in plants: discovering and tailoring genes to unlock the future. Curr. Opin. Biotechnol., 17: 113-122.

Wang L., Luo Y., Zhang L., Zhao J., Hu Z., Fan Y., Zhang C., 2008. Isolation and characterization of a C-repeat binding transcription factor from Maize. J. Integr. Plant Biol., 50(8): 965-974.

Wang X., Liu S., Liu X., Chen Z., Liu X., Pang Y., Sun X., Tang K., 2004. Molecular cloning and characterization of a $C B F$ gene from Capsella bursa-pastoris. DNA Seq., 15(3): 180-187.

Xiong Y., Fei S. Z., 2006. Functional and phylogenetic analysis of a DREB/CBFlike gene in perennial ryegrass (Lolium perenne L.). Planta, 224(4): 878-888. 
Yang S., Tang X. F., Ma N. N., Wang L. Y., Meng Q. W., 2011. Heterology expression of the sweet pepper $C B F 3$ gene confers elevated tolerance to chilling stress in transgenic tobacco. J. Plant Physiol., 168(15): 1804-1812.

Zhang X., Fowler S. G., Cheng H., Lou Y., Rhee S. Y., Stockinger E. J., Thomashow M. F., 2004. Freezing-sensitive tomato has a functional $\mathrm{CBF}$ cold response pathway, but a $\mathrm{CBF}$ regulon that differs from that of freezing-tolerant Arabidopsis. Plant J., 39(6): 905-919.

Zhuang L., Yuan X., Chen Y., Xu B., Yang Z., Huang B., 2015. PpCBF3 from coldtolerant kentucky bluegrass involved in freezing tolerance associated with upregulation of cold-related genes in transgenic Arabidopsis thaliana. PloS One, 10(7): e0132928. 